\title{
Encoding, repetition, and the mirror effect in recognition memory: Symmetry in motion
}

\author{
ANDREW HILFORD, MURRAY GLANZER, and KISOK KIM \\ New York University, New York, New York
}

\begin{abstract}
Attention/likelihood theory has been used to explain the mirror effect in recognition memory. The theory also predicts that any manipulation that affects the recognition of old items will also affect recognition of the new items-more specifically, that all the underlying distributions will move and that they will move symmetrically on the decision axis. In five experiments, we tested this prediction. The first two experiments used encoding tasks during study to change recognition performance for high- and low-frequency words. The results show symmetrical dispersion of the underlying distributions. The final three experiments used repetition to increase recognition performance. Repetition produced a symmetrical pattern of movement that was different from that produced by encoding task. This pattern is, however, also covered by attention/likelihood theory. A further extension of the theory was used to predict response times.
\end{abstract}

If there are two classes of items, with one class being better recognized than a second, then the first class is better recognized as old, when old, and also better recognized as new, when new. This regularity, which was identified by Glanzer and Adams (1985), is called the mirror effect. The mirror regularity can be produced using different item types-for example, low- and high-frequency words, with recognition memory for low-frequency words superior to that for high-frequency words (Gorman, 1961). When recognition for two such classes of items is tested, the results correspond to the positions of the distributions seen in Figure 1 (H and L refer to high- and lowfrequency items, respectively; $\mathrm{O}$ and $\mathrm{N}$ refer to old and new, respectively). The distributions in Figure 1 show the mirror pattern: The positions of the two new distributions mirror those of the old distributions.

Attention/likelihood theory has been introduced to explain the mirror effect (Glanzer \& Adams, 1990; Glanzer, Adams, Iverson, \& Kim, 1993; Kim \& Glanzer, 1993). It is a feature sampling theory that incorporates mechanisms of signal detection theory. The theory makes the following assumptions:

1. Stimuli consist of a set of features. The number of these features, $N$, is assumed to be equal for all stimuli. The marking of a feature indicates that this feature has been previously studied.

2. In all stimuli, some proportion of these features, $p$ (new), is already marked. This proportion corresponds

The research reported here was conducted in partial fulfillment of the doctoral degree by Andrew Hilford. It was supported by National Science Foundation Grant SBR-9409560. The authors thank Ted Coons, Lloyd Kaufman, Joe LeDoux, and Gay Snodgrass, members of the doctoral committee, for their helpful comments. Correspondence should be addressed to M. Glanzer, Department of Psychology, New York University, 6 Washington Place, New York, NY 10003 (e-mail: $\mathrm{mg} @$ xp.psych.nyu.edu). to a noise level. It is assumed to be constant for all new stimuli.

3. When a stimulus is studied, a random sample of features, $n(i)$, is examined by the participant. Different classes of stimuli elicit different amounts of attention from a participant, represented by different values of $n(i)$, with $i$ referring to a class of stimuli. These classes can be produced by selection (high- vs. low-frequency words) or experimental operations (efficient vs. inefficient encoding operations).

4. During study, all of the $n(i)$ features sampled are marked if they are not already marked. The proportion of features sampled is $\alpha(i)=n(i) / N$. Because some proportion of features, $p$ (new), is already marked before study (see Assumption 2), the state of stimuli in class $i$, after study, is given by the following equation:

$$
p(i, \text { old })=p(\text { new })+\alpha(i) \cdot[1-p(\text { new })] .
$$

A larger proportion of marking will occur under conditions in which participants engage in a more extensive examination of features. In such conditions, $\alpha(i)$ increases, and, as a result, discrimination increases.

5. During a recognition test, a participant also samples at random a set of features, $n(i)$, from a stimulus. It follows from the preceding statements that the number of marked features in the sample is binomially distributed with parameters $n(i)$ and $p(i$, old $)$ for old stimuli, and parameters $n(i)$ and $p$ (new) for new stimuli.

6. During the test, the participant checks each sampled feature to see whether it is marked. The participant counts the number of features that are marked. The participant then estimates how likely an old item is to have that amount of marking and how likely a new item is to have that amount of marking. These estimates are used to compute a likelihood ratio for that item, as presented in Equation 3. Decisions are based on the likelihood ratio, $\ln L(m \mid i, j)$, with 


$$
\begin{aligned}
\ln L(m \mid i, j) & =\ln \left[\frac{\left(\begin{array}{c}
n(i) \\
m
\end{array}\right) p(i, \text { old })^{\mathrm{m}} q(i, \text { old })^{n(i)-m}}{\left(\begin{array}{c}
n(i) \\
m
\end{array}\right) p(\text { new })^{m} q(\text { new })^{n(i)-m}}\right] \\
& =n(i) \ln \left[\frac{q(i, \text { old })}{q(\text { new })}\right]+m \ln \left[\frac{p(i, \text { old }) q(\text { new })}{p(\text { new }) q(i, \text { old })}\right]
\end{aligned}
$$

The values of $\ln L(m \mid i, j)$, known as $\lambda$, are binomially distributed:

$$
p[\ln L(m \mid i, j)=\lambda]=\left(\begin{array}{c}
n(i) \\
m
\end{array}\right) p(i, j)^{m} q(i, j)^{n(i)-m} .
$$

In the equations above, $m$ is the number of the features marked during sampling, and $i$ indicates the classes of items needed to produce the mirror effect (e.g., high- and low-frequency words). Finally, $j$ refers to the status of the stimulus, "old" or "new" as determined by whether the stimulus item appears in the study condition (old) or not (new). Despite the fact that $p$ (new) has the same value in both stimulus sets, when the distributions of the ratios, $\lambda$, described above, are plotted, the likelihood distributions for the new items of both classes separate from each other, giving the order seen in Figure 1. Detailed examples of computations and distributions are presented in Glanzer et al. (1993) and Kim and Glanzer (1993).

In the case of a two-alternative forced-choice test, the participant is viewed as comparing the $\lambda \mathrm{s}$ for the two test items and then choosing the one with the larger value. Up to now, the theory has been used to predict proportions of choice. An extension of the theory permits the prediction of response times (RTs) as well. This will be discussed after the prediction of the proportions of choice.

All types of recognition memory tests produce evidence of the mirror pattern (Glanzer \& Adams, 1985). One such test is the two-alternative forced-choice recognition test, used in all of the experiments reported here. The twoalternative forced-choice recognition test typically pre-

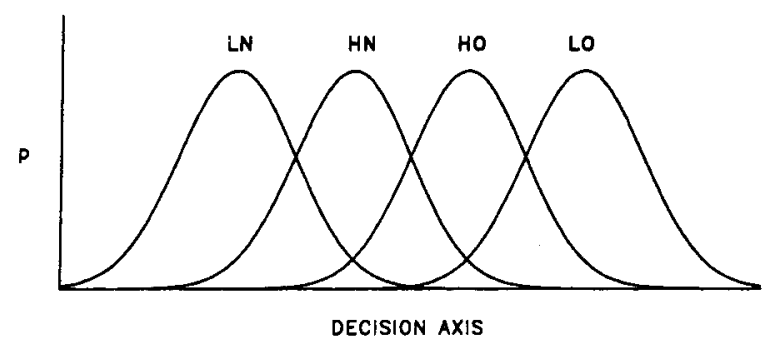

Figure 1. Underlying distributions displaying the mirror effect. $H$ and $L$ refer to the high- and low-frequency word classes, respectively; $O$ and $N$ refer to the conditions, old and new, respectively. The distributions in this figure, and those in Figures 2, 3, and 5 , are depicted as if they have equal variance. They do not. (For figures showing both variances and positions for a typical case according to attention/likelihood theory, see Kim \& Glanzer's, 1993, Figures 6 and 7). sents pairs of old $(\mathrm{O})$ and new $(\mathrm{N})$ items to the participants. The participants' task is to indicate which of the paired items is the old one-that is, the item seen in the study list. With two classes of items, L (low frequency, the superior class) and $\mathrm{H}$ (high frequency, the inferior class), there are ordinarily four tested pairs: $\mathrm{HO}, \mathrm{HN}$; $\mathrm{LO}, \mathrm{HN} ; \mathrm{HO}, \mathrm{LN}$; and LO,LN. These four pairs that match old words with new words are called here standard choice pairs. The recognition tests used here include two additional test pairs introduced by Glanzer and Bowles (1976). The new pairs consist of either two old items, one from each class, LO,HO, or two new items, one from each class, HN,LN. These two pairs are called null choice pairs because there is no obvious reason to choose either item of the pair. The null choice pairs are useful in that they give further information regarding the positions of the underlying distributions along the decision axis--in particular, the relation of $\mathrm{LO}$ to $\mathrm{HO}$ and the relation of $\mathrm{HN}$ to $\mathrm{LN}$.

When the mirror effect holds, as in Figure 1, the following relations hold in the recognition performance for the six forced-choice pairs. For the standard choices,

$P(\mathrm{HO}, \mathrm{HN})<P(\mathrm{LO}, \mathrm{HN}), P(\mathrm{HO}, \mathrm{LN})<P(\mathrm{LO}, \mathrm{LN})$

and for the null choices,

$$
P(\mathrm{HN}, \mathrm{LN}), P(\mathrm{LO}, \mathrm{HO})>.50,
$$

where $P$ refers to the proportion of choice of the first argument of the pair over the second. For the standard choice pairs, the overall set of inequalities represents recognition accuracy. For the null pairs, $P(\mathrm{HN}, \mathrm{LN})$ and $P(\mathrm{LO}, \mathrm{HO})$, the inequalities indicate the position of $\mathrm{HN}$ with respect to $\mathrm{LN}$ and of $\mathrm{LO}$ with respect to HO. They are therefore also useful in analyzing the movement of the distributions, when an experimental operation changes the distances between the two old distributions and between the two new distributions.

Attention/likelihood theory not only predicts the presence of the mirror effect but it also predicts the following: Conditions that impair or hinder recognition performance cause a symmetric movement of both the old and the new underlying distributions. A formal proof supporting this assertion may be found in Glanzer, Adams, and Iverson (1991). Previous experiments have shown that decreasing study time (Kim \& Glanzer, 1993) or increasing the time between study and test (Glanzer et al., 1991) caused the distributions to collapse toward the midpoint of the decision axis. All four distributions moved toward a central point on the decision axis, while maintaining the mirror pattern. In those cases, the movement as evidenced by changes in the proportions of choices not only decreased the distance of each underlying distribution from a central point on the decision axis but also decreased the distance of each distribution from its neighbor. This pattern of movement is shown in Figure 2. A different pattern of symmetric movement can also occur with a decrease in accuracy. Such a pattern was found by Kim and Glanzer (1995) in which output interference caused the inner distributions ( $\mathrm{HN}$ and $\mathrm{HO}$ ) to move farther toward the center 

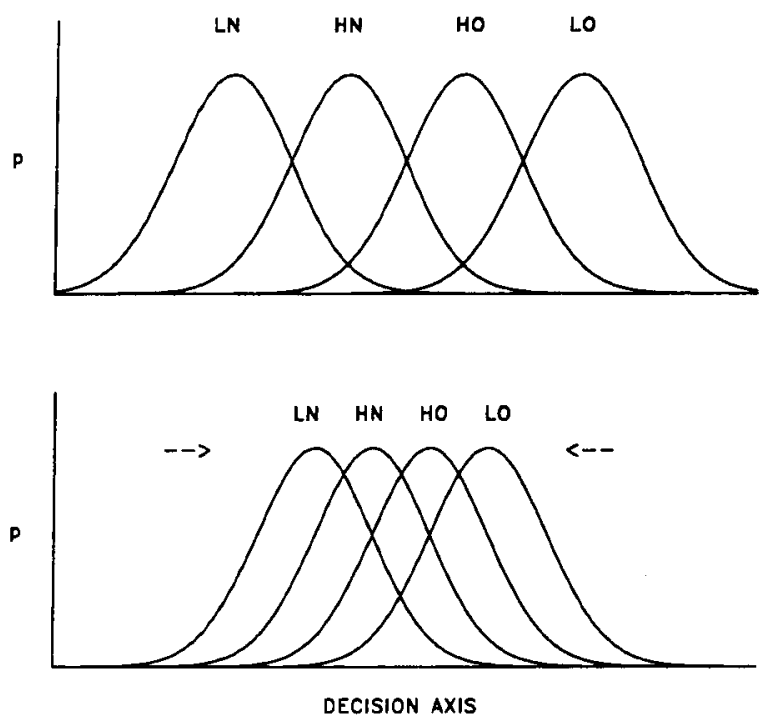

Figure 2. Schematization of the pattern labeled concentering (Glanzer et al., 1993) produced by a decrease in recognition performance. The top panel shows the initial position of the underlying distributions. The bottom panel shows them moving to a central point and closing up on each other as accuracy decreases.

than the outer distributions ( $\mathrm{LN}$ and LO). Both patterns of symmetric, centripetal movement can be covered by attention/likelihood theory.

According to attention/likelihood theory, the symmetric, centripetal movement of the underlying distributions can occur in a number of ways. One way is by a reduction in sampling and marking of features. This occurs when study time is reduced. Another way is by loss of previously marked features. This occurs with forgetting. The smaller numbers of marked features alter the likelihood ratios so that the distances between the distributions decrease and the proportions of choice are lower. It follows from this that if manipulations that reduce feature marking produce a symmetric movement of the four distributions toward the center point, then manipulations that increase feature marking should produce a symmetric movement of the distributions away from the central point. Increases in feature sampling and marking should result in an increase in recognition performance and changes in the positions of the underlying distributions on both sides of the decision axis. The changes we expected are represented in Figure 3. Figure 3 shows the reverse of the movement in Figure 2. Evidence of the centrifugal movement in Figure 3 would be increases in all the proportions of choice in Inequalities 4 and 5 listed earlier, both for the standard choices in Inequality 4 and for the null choices in Inequality 5.

The experiments reported here attempted to produce a symmetric centrifugal pattern of movement by means of two variables: by varying encoding tasks, and by repetition. Both variables were expected to increase the marking of features and consequently improve recognition performance. The encoding variable (Experiments 1 and 2) produces the pattern of movement schematized in Fig- ure 3. Repetition (Experiments 3, 4, and 5) produces a different, although symmetric, pattern of movement.

\section{DISPERSING THE UNDERLYING DISTRIBUTIONS BY VARYING THE ENCODING TASK}

\section{Experiment 1}

In Experiment 1, two encoding tasks were used to produce different levels of recognition performance. One group of participants was given a less effective encoding task during the study phase (letter counting), and the second group was given a more effective encoding task (frequency judgment). Both groups were expected to produce the mirror pattern with the underlying distributions in the second group farther from the center of the decision axis and from each other.

\section{Method}

The participants were divided into two groups. One group counted the letters in each of the words of the study list. The other group judged the frequency of the study words. Both groups were tested after completing the study list. Both the study list and the test list were individually randomized for each participant so that each participant was given a unique pair of lists. This holds for all the experiments that follow.

Two independent variables were used: encoding task (between participants) and word frequency (within participants). The encoding tasks were letter counting and frequency judgment. On the basis of past work (Craik \& Tulving, 1975) frequency judgment was expected to be more effective than letter counting. Word frequency, randomly mixed in the study list, was used to produce the two classes of items needed to show the mirror pattern. This also holds for the rest of the experiments in this paper.

Participants. Seventy-two undergraduate students from an introductory psychology class took part in the experiment to fulfill a
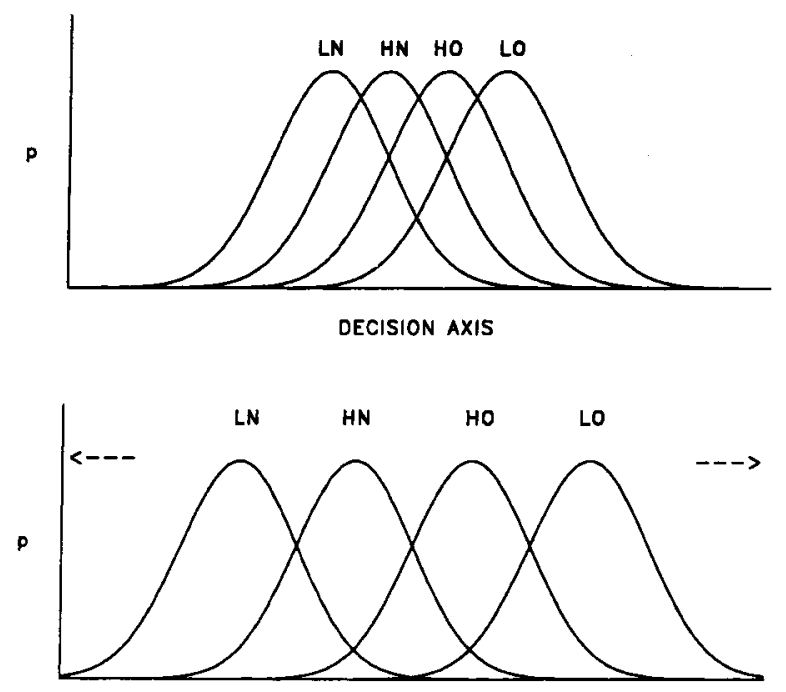

Figure 3. Schematization of the dispersion of the underlying distributions expected as a result of increasing recognition performance. The top panel shows the initial position of the underlying distributions. The bottom panel shows their dispersion in an increased learning condition. This pattern is produced by increasing accuracy through encoding task. 
course requirement. All of the participants had been speaking English since the age of 9 or earlier. The description of the participants here holds for the participants in all of the experiments that follow.

Procedure. The participants were randomly assigned to the two encoding conditions. In the letter counting condition, the participants were shown a list of 180 study words, and they judged the number of letters in each. If the words contained seven or more letters, they pressed a key marked "YES." If the word contained fewer than seven letters, they pressed a key marked "NO." In the frequency judgment condition, the participants viewed 180 words and decided whether each word was a common word or an uncommon word. If they thought the word common, they pressed the "YES" key; if not, they pressed the "NO" key. The words were presented one at a time, centered, in uppercase letters on a monochrome computer monitor. The presentation time for each word was $2,000 \mathrm{msec}$, with a 250 msec interstimulus interval (ISI). The experiment was run on an IBM-compatible computer using MEL software (Schneider, 1988). The study lists started and ended with five randomly selected filler items, which were used to eliminate recency and primacy effects.

Following the completion of the study list, the participants were given a two-alternative forced-choice recognition test. The recognition test consisted of 180 word pairs with equal numbers ( 30 pairs) of the two null choice pairs (HN,LN and $\mathrm{LO}, \mathrm{HO}$ ) and the four standard choice pairs (HO,HN; LO,HN; HO,LN; and LO,LN). One pair appeared at a time and remained on the screen until the participant responded. The pairs appeared one word above the other, with position counterbalanced so that in one half of the presentations the preferred choice occupied the top position. The participants were instructed to choose the word of the pair that had appeared in the preceding study list. The participants were also told to make a bestguess choice when they could not decide on a word in any pair. They recorded their choices by pressing one of two marked keys: one with a $\uparrow$, and the other, below it, with a $\downarrow$. The procedure for the recognition test holds for the rest of the experiments in this paper. Each test list began with five filler pairs, which were not included in analysis.

The participants were given a 10 -word practice study list and a test list of 10 forced-choice pairs at the start of the experiment. The practice study list was given with the same encoding task as the main list that followed.

Materials. Three hundred sixty words were selected from Kučera and Francis (1967). The words were chosen so that half were highfrequency words (40 or more times per million) and half were lowfrequency words ( 8 or fewer times per million). The two frequency sets were matched for word length and concreteness, with concreteness measures based on the Paivio, Yuille, and Madigan (1968) norms. The 90 high-frequency words and the 90 low-frequency words for the study list were selected at random independently for each participant. They were also ordered randomly for each participant. The assignment of words to test pairs was also done randomly for each participant, with the restriction that there be 30 pairs in each test condition. The test pairs were ordered randomly, independently for each participant's test list. This description of the construc- tion of study and test lists by independent, random selection and random ordering of items holds for all the experiments that follow.

\section{Results}

The results of Experiment 1 are summarized in Table 1. The column headings refer to each of the six forced-choice pairs in the recognition test. The rows contain the results of the two encoding groups for both the observed and predicted conditions. The observed means summarize the actual performance in each forced-choice condition, averaged across the 36 participants in each group. The $M S_{\mathrm{e}}=0.011$. The predicted means (discussed later) showing the results of fitting the theory to the data are listed below the observed means. Also, at the bottom of the table are the parameters used for fitting. Conventional analyses of variance (ANOVAs) were carried out on all the experiments in this report. The results for all five experiments are the same. Statistically significant effects of the main variable (here, encoding) and a statistically significant interaction of that variable with choice condition (LO,LN; HO,LN; etc.).

There are two aspects of the data that we focus on: (1) the presence of the mirror pattern as evidenced by the inequalities in the proportions of choice for the standard conditions (Inequality 4, given earlier) and the inequalities for the null conditions (Inequality 5), and (2) the movement of the underlying distributions as evidenced by the increases in the proportions of choice both in the standard conditions and in the null conditions.

The mirror pattern is seen here in both encoding conditions

$$
P(\mathrm{HO}, \mathrm{HN})<P(\mathrm{LO}, \mathrm{HN}), P(\mathrm{HO}, \mathrm{LN})<P(\mathrm{LO}, \mathrm{LN})
$$

and

$$
P(\mathrm{HN}, \mathrm{LN}), P(\mathrm{LO}, \mathrm{HO})>.50 .
$$

The tie between $P(\mathrm{HO}, \mathrm{HN})$ and $P(\mathrm{LO}, \mathrm{HN})$ in the frequency judgment condition appears to depart from the pattern. Actually, there was a slight difference between the two pairs, with $P(\mathrm{HO}, \mathrm{HN})<P(\mathrm{LO}, \mathrm{HN})$, as expected, but the difference was lost after rounding. We will, however, consider this departure further.

The data, moreover, show that the two encoding tasks produced two different levels of recognition performance, with frequency judgment yielding, as expected, a higher overall performance than letter counting. In Table 1, the

Table 1

Mean Proportions of Choice for the Letter Counting and

\begin{tabular}{|c|c|c|c|c|c|c|}
\hline \multirow[b]{2}{*}{ Condition } & \multicolumn{2}{|c|}{ Null Pairs } & \multicolumn{4}{|c|}{ Standard Pairs } \\
\hline & $P(\mathrm{HN}, \mathrm{LN})$ & $P(\mathrm{LO}, \mathrm{HO})$ & $P(\mathrm{HO}, \mathrm{HN})$ & $P(\mathrm{LO}, \mathrm{HN})$ & $P(\mathrm{HO}, \mathrm{LN})$ & $P(\mathrm{LO}, \mathrm{LN})$ \\
\hline \multicolumn{7}{|l|}{ Letter Counting } \\
\hline Observed & .62 & .54 & .71 & .74 & .75 & .80 \\
\hline Predicted & .59 & .56 & .72 & .75 & .77 & .79 \\
\hline \multicolumn{7}{|c|}{ Frequency Judgment } \\
\hline Observed & .66 & .55 & .85 & .85 & .88 & .90 \\
\hline Predicted & .65 & .61 & .84 & .87 & .88 & .90 \\
\hline
\end{tabular}
Frequency Judgment Encoding Task Groups in Experiment 1

Note Parameters: $p($ new $)=.0827 ; N=1,000$ (preset $) ; n$ (high) $=61 ; n($ low $)=74 ; \gamma=.5850$.

$r^{2}=.97$. There were 36 participants in each condition. 
proportions of choice values are higher in each of the choice pairs in the frequency judgment group than in the letter counting group.

Finally, and most important, the fact that all the proportions in the frequency judgment condition are greater than the corresponding proportions in the letter counting condition shows that the distributions in the frequency judgment condition are farther apart from each other than the same distributions in the letter counting condition. This was the pattern schematized in Figure 3 that we expected on the basis of preceding work (Glanzer et al., 1993; Kim \& Glanzer, 1993). The overall change in the null conditions as a function of encoding task was, however, only borderline in statistical significance $[t(350)=1.53$, $p<.07$, one-tail].

\section{Discussion}

Given the two levels of recognition performance, attention/likelihood theory predicts the following: (1) the distributions in both conditions will show the mirror pattern, which they do, and (2) changes that affect the positions of the old distributions will also affect the positions of the new distributions, which they also do.

The result of fitting attention/likelihood theory to the data appears in Table 1, in the rows labeled "Predicted." These predicted values are based on the parameters listed at the bottom of the table. $N$ represents the total number of features contained in an item. $N$ was set beforehand at 1,000 on the basis of earlier work, whereas the rest of the parameters were allowed to vary. Two attention parameters, $n$ (high) and $n$ (low), were used to account for the differential feature sampling for the two word frequency classes. The third parameter $p$ (new) represents the noise level for all words at the beginning of the experiment. A parameter, $\gamma$, was included to reduce $n(i)$ for letter counting, the less effective encoding condition. This last parameter was set with $0<\gamma<1$. With $N$ set beforehand, four parameters were used to predict the 12 observed means.

The values for the parameters were determined by fitting the equations of attention/likelihood theory to the data using the function-minimizing program PRAXIS (Brent, 1973). The $C$ version, which was used in this study, was written by Gegenfurtner (1992).

The fitting was accomplished by minimizing the value of

$$
\sum_{k=1}^{m} \frac{[\hat{P}(k)-P(k)]^{2}}{[\hat{P}(k)] \cdot[1-\hat{P}(k)]},
$$

where $k$ is the choice condition (e.g., the comparison of $\mathrm{HN}$ and $\mathrm{LN}$ in the letter counting encoding condition), $\hat{P}(k)$ is the theoretical proportion, $P(k)$ is the observed proportion, and $m$ is 12 (the number of proportions corresponding to the six choice conditions in each of the two encoding task groups). This fitting procedure was also used in Experiments 2, 3, 4, and 5. When the observed values are regressed on the predicted values we obtain an $r^{2}$ of .97 , indicating that $97 \%$ of the variance was accounted for. Although the pattern of inequalities and the changes in Table 1 conformed to our expectations and were fitted well by the theory, there were two aspects of the data that were not completely satisfactory. One was the borderline statistical effect of the changes in the null conditions. The other was the equality of $P(\mathrm{HO}, \mathrm{HN})$ and $P(\mathrm{LO}, \mathrm{HN})$ in the frequency judgment condition. We thought that both of these may have resulted from the characteristics of the frequency judgment task. In that encoding task, the participants said "yes" to high-frequency old words and "no" to low-frequency old words. If "yes" responses produce a carryover bias toward selecting a word as old in a recognition test and "no" responses produce a bias against selecting a word as old, then $P(\mathrm{LO}, \mathrm{HO})$ would be lower than it would be without such bias. Also, $P(\mathrm{LO}, \mathrm{HN})$ would be lower. The data of Craik and Tulving (1975) support the idea that such a carryover bias from encoding task to memory exists--particularly, in semantic encoding tasks. We therefore decided to replicate the experiment with a change in the more effective encoding task. We made use of an encoding task that produced the same response to high- and low-frequency words. In this way, carryover bias would be eliminated.

Before we leave Experiment 1, we note that the theoretical parameters computed to predict the proportions of choice give us the basis for predicting the RTs. Corresponding to the 12 mean proportions of choice in Table 1 are 24 RTs: 12 for the correct responses and 12 for the incorrect responses.

To relate the mean log likelihood values to the RTs, we extend the linear equation presented by Piéron (1914, $1920)$ relating RTs to stimulus intensity:

$$
\log \left(\mathrm{RT}-r_{o}\right)=\log k-\beta \log i,
$$

where $r_{o}$ is some minimal processing time, $\log k$ is the intercept, $\beta$ is the slope, and $i$ is a measure of intensity. To extend the equation to our data, we introduce two changes. First, we replace intensity with $\log$ likelihood ratios, $\lambda$, the equivalent of intensity in our formulation. Second, we allow for the effect of two variables: the size of the log likelihood ratio for the selected alternative (the equivalent of intensity), and, since we are dealing with a comparison between two alternatives, the difference between the likelihood ratio of the selected and the unselected alternative. The equation for the correct responses $(C)$ becomes:

$$
\begin{aligned}
\ln \left[\operatorname{RT}(C)-r_{o}\right)= & a-b_{1}(M(\lambda \mid S, C)] \\
& -b_{2}[M(\lambda \mid S, C)-M(\lambda \mid U, C)]
\end{aligned}
$$

The equation states that the greater the mean log likelihood ratio of the selected item $(S)$ and the greater the difference between that and the mean log ratio of the unselected item $(U)$, the less time it takes the participant (beyond some minimal processing time, $r_{o}$ ) to make a decision. For incorrect responses, the argument $C$ (for correct) is replaced by $I$ (for incorrect). The parameters $b_{1}$ and $b_{2}$ are the regression weights, and $a$ is the intercept.

Application of this regression equation to the RTs of Experiment 1 permits us to account for $91 \%$ of the RT variance in the letter encoding condition and $88 \%$ of the variance in the frequency condition group. The mean re- 
lations between the observed RTs and the predicted RTs are shown in Figure 4 for both encoding conditions. RTs below $500 \mathrm{msec}$ and above $5,000 \mathrm{msec}$ were removed before computing mean observed RTs. The $M S_{\mathrm{e}}$ for the ln $\mathrm{RT}=0.0189$. The fitting of RTs was carried out for the remaining experiments. Overall, the approach accounted for an average of $87 \%$ of the RT means. The fitting of RTs will, however, not be considered further. Details concerning the computation and the fit of other RT data may be obtained by writing to the authors.

\section{Experiment 2}

Experiment 2 was conducted to replicate and extend the findings of Experiment 1. We replaced the frequency judgment task with a lexical decision task, another semantic level encoding task. In lexical decision, participants view a series of words and nonwords and indicate which
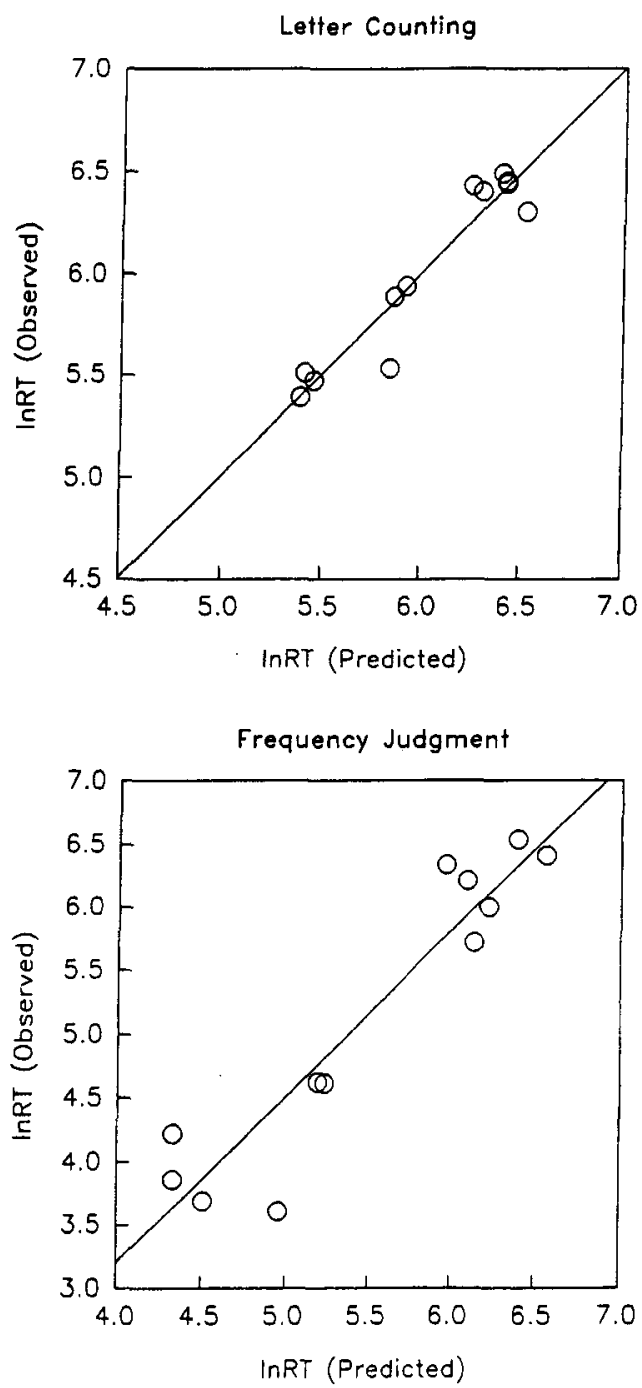

Figure 4. Plots relating predicted to observed correct and incorrect response times for the two encoding conditions in Experiment 1. are words. In this task, participants treat low- and highfrequency words the same way in carrying out the encoding task. They say "yes" to both. The carryover bias that may have distorted the data of Experiment 1 would therefore be eliminated.

\section{Method}

The method was the same as in Experiment 1 except that half of the participants were given a lexical decision encoding task instead of the frequency judgment task. They viewed an equal number of words and nonwords and indicated whether or not they thought each item was an English word. The other participants did letter counting as in Experiment 1, but on both words and nonwords. Only the words appeared in the recognition test. The design and procedure was the same in Experiment 1, except for the substitution of lexical decision for frequency judgment as the more effective encoding task.

Participants. Seventy-two undergraduate students participated.

Procedure. The participants were randomly assigned to one of the two encoding tasks. Those given the letter counting test viewed a list of words and nonwords (to match the study list length with the lexical decision group) and indicated with the "YES" or "NO" key whether the items contained seven or more letters. The participants given the lexical decision task viewed the same lists of words and nonwords, and they were instructed to determine whether or not each item was a word. If they thought an item was an actual word, they pressed the key marked "YES"; if not, they pressed the key marked "NO." The ISI for the items was $250 \mathrm{msec}$, and the item presentation duration was $1,500 \mathrm{msec}$.

All of the participants were given a two-alternative forced-choice recognition test following the completion of the study section. The test was structured and presented in the same way as in Experiment 1.

Materials. The study and test lists were created from the same word pool used for the lists in Experiment 1, with 180 nonwords added for the study lists. The nonwords were created by taking words and changing one or more letters until the item was no longer a word but was legal in appearance (did not violate English orthographic rules). The 180 words and 180 nonwords were put in mixed random order, each list randomly ordered independently for each participant.

\section{Results}

The results are summarized in Table 2 . The rows labeled "Observed" show the proportions of choice for each forced-choice pair, averaged for the 36 participants in each condition. The $M S_{\mathrm{e}}$ for these means $=0.011$. The corresponding predicted values based on the theory appear in the rows labeled "predicted." The values of the parameters for the fitting of the theory to the data appear at the bottom of the table.

A full mirror pattern is present in both the letter counting and the lexical decision group conditions with the expected series of inequalities. In addition, the lexical decision task was effective in increasing encoding over the letter counting task. In each of the six forced-choice pairs, the proportion of choice is higher in the lexical decision condition than the corresponding value in the letter counting condition. Test of the overall change in the null conditions showed a statistically significant effect $[t(350)=$ $2.63, p<.005$, one-tail].

The increase in the proportions of choice in the lexical decision group for both the standard choices and the null choices indicates clearly that the underlying distributions for this group moved away from the center of the 
Table 2

Mean Proportions of Choice for the Lexical Decision and

Letter Counting Encoding Task Groups in Experiment 2

\begin{tabular}{|c|c|c|c|c|c|c|}
\hline \multirow[b]{2}{*}{ Condition } & \multicolumn{2}{|c|}{ Null Pairs } & \multicolumn{4}{|c|}{ Standard Pairs } \\
\hline & $P(\mathrm{HN}, \mathrm{LN})$ & $P(\mathrm{LO}, \mathrm{HO})$ & $P(\mathrm{HO}, \mathrm{HN})$ & $P(\mathrm{LO}, \mathrm{HN})$ & $P(\mathrm{HO}, \mathrm{LN})$ & $P(\mathrm{LO}, \mathrm{LN})$ \\
\hline \multicolumn{7}{|c|}{ Letter Counting } \\
\hline Observed & .55 & .59 & .66 & .69 & .69 & .71 \\
\hline Predicted & .57 & .57 & .66 & .70 & .70 & .73 \\
\hline \multicolumn{7}{|c|}{ Lexical Decision } \\
\hline Observed & .59 & .64 & .71 & .80 & .73 & .83 \\
\hline Predicted & .61 & .62 & .73 & .79 & .77 & .82 \\
\hline
\end{tabular}

decision axis and from each other. Figure 3 schematizes the positions for the two experimental conditions, with letter counting represented in the top panel and lexical decision represented in the bottom panel.

\section{Discussion}

Experiment 2 replicated and extended the results of Experiment 1 . Increasing encoding efficiency produces not just an improvement in recognition performance but a specific pattern of improvement. Recognition performance increases in the more effective encoding task condition across all six forced-choice pairs, while maintaining the pattern of inequalities of the mirror effect. Moreover, both the new and the old distributions pull away from the center of the decision axis, and, at the same time, the distances between $\mathrm{LN}$ and $\mathrm{HN}$ and between $\mathrm{LO}$ and $\mathrm{HO}$ also increase. It is important to note that although the two new distributions move along the decision axis, neither encoding task directly affected these items. The new words were presented in neither the letter counting condition nor the lexical decision condition.

Fitting was carried out as in Experiment 1, using four parameters for the 12 observed means. $N$ was again fixed beforehand at 1,000 . The fit of the theory to these data is satisfactory $\left(r^{2}=.95\right)$.

Having produced a symmetrical dispersion of the underlying distributions by varying encoding tasks, we decided to extend the findings further by attempting to obtain similar results with a different manipulation. The remaining experiments used repetition of list items as a means of increasing recognition performance. While increasing encoding effectiveness allowed for a greater degree of feature sampling and marking during a single trial, repetition was expected to produce the same outcome, but by providing a second opportunity to sample and mark features. The results show that this manipulation produced a different pattern of symmetrical movement.

\section{DISPERSING THE UNDERLYING DISTRIBUTIONS BY REPETITION}

\section{Experiment 3}

In Experiment 3, participants viewed a list of high- and low-frequency words either once or twice. Repetition was expected to increase recognition performance and pull apart the underlying distributions, as did the use of a more effective encoding task in Experiments 1 and 2.

\section{Method}

The participants were randomly assigned to one of two groups. Both groups performed a lexical decision task on the study list. One group, however, did this once, whereas the other repeated the task. Both groups were given a surprise two-alternative forced-choice recognition test following the completion of the study list. The main independent variable was single versus repeated item presentation, which was a between-group variable. The second independent variable was word frequency, which was a within-group variable. Twenty-four participants were randomly assigned to each of the two study conditions.

Participants. Forty-eight undergraduate students participated

Procedure. The participants were first presented with a selfpaced lexical decision study task like that in Experiment 2. The participants in the single-presentation condition viewed and judged each item once. In the repetition condition, the same study list was rerandomized, and the task was repeated in a second presentation. The participants were then given a surprise two-alternative forcedchoice recognition test presented as in the preceding experiments.

The study lists contained an equal number of words (180) and nonwords (180), with the words divided equally into high- and lowfrequency words. The study lists started with nine filler items and ended with five filler items.

The participants were instructed to respond as quickly and accurately as possible. Although the presentation was self-paced, feedback was given during the lexical decision task to maintain speed. The feedback that followed each response was in the form of the numbers 1 through 9 , each number referring to the relative speed of the response. The number " 1 " on the screen indicated a response of $750 \mathrm{msec}$ or less, "2" a response of 751-1,000 msec, and so on.

Materials. The study and test lists were constructed in the same way as those in Experiment 2. For the repetition condition, the 360 item lists ( 180 words, 180 nonwords) were rerandomized into new lists and presented a second time.

\section{Results}

The results of Experiment 3 are summarized in Table 3. The mean proportions of choices for each experimental condition appear in the two rows labeled "Observed." The $M S_{\mathrm{e}}$ for these means $=0.010$.

The mirror pattern appears in both the single- and the repeated-presentation conditions, with repetition producing an average increase in the proportions. Here, however, in contrast with Experiments 1 and 2, the standard comparisons are affected by the main variable, but the null comparisons are not. This means that the pattern of movement seen in Figure 3 does not hold for these data. 
Table 3

Mean Proportions of Choice for the Single- and

Repeated-Presentation Groups in Experiment 3

\begin{tabular}{|c|c|c|c|c|c|c|}
\hline \multirow[b]{2}{*}{ Condition } & \multicolumn{2}{|c|}{ Null Pairs } & \multicolumn{4}{|c|}{ Standard Pairs } \\
\hline & $P(\mathrm{HN}, \mathrm{LN})$ & $P(\mathrm{LO}, \mathrm{HO})$ & $P(\mathrm{HO}, \mathrm{HN})$ & $P(\mathrm{LO}, \mathrm{HN})$ & $P(\mathrm{HO}, \mathrm{LN})$ & $P(\mathrm{LO}, \mathrm{LN})$ \\
\hline \multicolumn{7}{|l|}{ Single } \\
\hline Observed & .62 & .57 & .70 & .77 & .74 & .78 \\
\hline Predicted & .60 & .59 & .72 & .76 & .76 & .79 \\
\hline \multicolumn{7}{|l|}{ Repeated } \\
\hline Observed & .60 & .59 & .80 & .86 & .84 & .88 \\
\hline Predicted & .60 & .61 & .81 & .86 & .84 & .88 \\
\hline
\end{tabular}

Note-Parameters: $p$ (new) $=.09 ; N=1,000$ (preset); $n$ (high, 1$)=43 ; n($ low, 1$)=55$; $n($ high, 2$)=27 ; n($ low, 2$)=28 ; r^{2}=.99$. There were 24 participants in each condition.

In the preceding experiments, schematized in Figure 3, the more effective encoding condition increased the distances between all four underlying distributions. Here, the increase does not affect all of the distances. The proportions of choice are higher only in the four standard choice conditions of the repetition condition, indicating that, after repetition, the participants were better able to distinguish old words from new words. But the null choice pairs were unaffected. Only a slight increase (.02) from the single to the repeated conditions is seen in the old null pair condition, LO,HO; the new null pair condition, $H N, L N$, exhibits a reversal, with the proportion of choice in the repetition condition less (by .02) than that in the single-presentation condition. There is therefore no overall change in the null conditions.

\section{Discussion}

Our initial expectation was that repetition would act in a manner similar to that of increasing encoding effectiveness: increases in recognition performance with dispersion of the underlying distributions away from the center point of the decision axis and also away from each other. The data show a different pattern of movement. They show that although the pair of old distributions separated from the pair of new distributions, the two old distributions $\mathrm{LO}$ and $\mathrm{HO}$ did not separate from each other, and the two new distributions HN and LN did not separate from each other. Figure 5 schematizes the results of Experiment 3 . It shows a symmetry movement different from that found in Experiments 1 and 2.

The top panel of Figure 5 illustrates the positions of the four distributions after a single presentation of the study items. This is a standard mirror pattern. The horizontal bars represent the distances separating the distributions $\mathrm{HO}$ and $\mathrm{LO}$ and separating $\mathrm{LN}$ and $\mathrm{HN}$. The bottom panel sılows the pattern of changes seen in these data. The increase in distance between the two old and two new distributions is represented by the raised horizontal bar. The lower horizontal bars are approximately the same across the two panels, showing that the distance separating LO from $\mathrm{HO}$ and $\mathrm{HN}$ from LN after repetition is about the same as that of the single-presentation condition. This pattern of movement can, however, as we will show, be covered by the theory.
We considered the possible reasons for the pattern produced in this study and what it indicated about the way the theory should be applied. An analysis of the RTs in the lexical decision task showed that the participants did not respond in the same way to the two frequency classes across the two presentations. Table 4 shows that the participants spent more time on the low-frequency words than on the high-frequency words when the words appeared the first time. However, with repetition, the RTs dropped for the low-frequency words but not for the high-frequency words. The changes in the RTs suggest that the participants reduced the time spent processing the low-frequency words when they appeared a second time. This change could account for the pattern produced by repetition. The reduction in processing of the low-frequency words during repetition would lessen the effect on the low-frequency words of the second trial and work against the separation of LO from $\mathrm{HO}$ and $\mathrm{LN}$ from $\mathrm{HN}$.

There are other studies that show a pattern of RT changes to low-frequency words as contrasted with high-



Figure 5. Schematization of the dispersion of the underlying distributions by repetition for single presentation (top panel) and repetition (bottom panel). 
Table 4

Mean RTs (in Milliseconds) in the First and Second Trials of the Repetition Condition Lexical Decision Tasks for High- and Low-Frequency Words in Experiment 3

\begin{tabular}{lcc} 
& \multicolumn{2}{c}{ Word Frequency } \\
\cline { 2 - 3 } Trial & High & Low \\
\hline First & 558 & 613 \\
Second & 569 & 594 \\
\hline
\end{tabular}

frequency words similar to that in Table 4. Scarborough, Cortese, and Scarborough (1977) presented participants with a lexical decision task, repeating high- and lowfrequency words. Their RTs to both high-frequency and low-frequency words dropped across the two presentations, but the drop was greater for the low-frequency words. The frequency $\times$ presentation interaction was statistically significant $(p<.05)$. Forbach, Stanners, and Hochhaus (1974) found similar changes in RTs for highand low-frequency words for single and repeated trials in a lexical decision task. In our own RT data cited above, the interaction of frequency with trials in the repetition group was statistically significant $(p<.004)$.

The difference in change of RTs to the two word classes indicates that participants reduce their processing of lowfrequency words on a second trial more than they do the processing of high-frequency words. These changes can be incorporated in the theory by allowing the attention parameter to change on the second trial and permitting the change to differ for the two frequency conditions. The parameter $n(i)$ was therefore rewritten as $n(i, t)$, where $t$ refers to the trial. This addition allowed $n(i)$ to move independently across the two trials for the high-and lowfrequency word classes. The theory, extended in this way, was fitted to the 12 observed means in Table 3 . The results of the fitting, given in Table 3 , show that on the first trial, $n$ (high, 1 ) is much lower than $n$ (low, 1 ), which is what would be expected: The low-frequency items draw more attention than the high-frequency words. The attention parameter for the less common words should, therefore, be higher than for the more common words. However, during the second trial, while both parameters drop, the low-frequency attention parameter $n($ low,2) drops much farther than the high-frequency parameter $n$ (high,2). In fact, the two attention parameters are nearly equal. This corresponds to the changes in RT data and shows that second presentation does not benefit the low-frequency words as much as the first presentation. Using five free parameters for the 12 observed means, the fit is $\operatorname{good}\left(r^{2}=.99\right)$.

We interpret the data as showing movement of both old and new distributions from the center of the decision axis, with the two old distributions moving the same distance to the right and the two new distributions also moving the same distance to the left. An alternative interpretation is that only the old distributions move while the new distributions stay fixed in both the single and repeat conditions. With only the old distributions moving, the standard choice pairs would increase with repetition, while the null choice pairs would remain constant. There is an argument against this interpretation. Movement on both sides of the decision axis has already been established in a large number of experiments (Glanzer et al., 1993), as well as in the two preceding experiments. In those experiments, both the old and the new distributions were seen under a variety of manipulations to move with respect to the center point of the decision axis. The six experiments summarized in Glanzer et al. (1993) all show bilateral, symmetric movement of the underlying distributions. They support the idea that the new and the old distributions move. To argue for fixedness of the new distributions for the present data would require that some reason or theory be given to separate these findings from the findings of eight closely related experiments.

In summary, the data from Experiment 3 can be covered by attention/likelihood theory. The pattern of movement of the underlying distributions is different from that found with encoding differences, but it is still symmetrical. The fitting, however, requires an increase in the number of estimated parameters from four, as in Experiments 1 and 2, to five. The experiments that follow were designed to determine whether a pattern of movement such as that seen in Experiments 1 and 2, with the increase of all distances, could be obtained with repetition or if pattern of movement in the old and new distributions seen here is consistently produced by repetition.

\section{Experiment 4}

Before settling on the interpretation offered for the data of Experiment 3 involving a differential change in attention, we tried to rule out other factors that might have produced the pattern of movement we found. One was a possible ceiling effect that limited the movement of the underlying distributions--particularly, those of the lowfrequency words. In Experiment 4, we made two changes, both designed to eliminate ceiling effects. First, we presented each item in the study list for a fixed duration rather than presenting them self-paced by the participants (as in Experiment 3). Second, we replaced the lexical decision, which is a very effective encoding task, with a simple instruction to study. We also changed to a within-group experimental design in order to reduce variability.

\section{Method}

The participants were presented four study-test blocks. In two of these blocks, each word appeared once in the study list, in the other two, each word appeared twice. The experiment was run in an ABBA and $\mathrm{BAAB}$ design, to counterbalance order effects of the blocks. Half of the participants were assigned randomly to each block order. Following each study list, the participants were given a twoalternative forced-choice recognition test. The independent variables were repetition and word frequency. Both of these were withingroup variables.

Participants. Twenty-four undergraduate students participated.

Procedure. Study list items were presented on the screen one word at a time. The participants were instructed to study the words as they appeared and told that they would be tested on them afterwards. The participants were informed that some of the words might be repeated. 
The study words were presented for $750 \mathrm{msec}$, with a $250-\mathrm{msec}$ ISI. The recognition test was self-paced. The experiment started with a short study-and-test practice session. The practice study list contained 20 words, and the practice test contained 20 two-alternative forced-choice pairs.

Materials. Two hundred forty high-frequency words and 240 low-frequency words were used. Each study list contained 60 words made up of equal numbers of high- and low-frequency words. Each repeated study list was created by duplicating a list of 60 words and then randomizing the new list of 120 words. Each test list consisted of 60 pairs- 10 pairs in each of the four standard and two null choice pairs. The study lists were preceded by 5 filler items and followed by 5 filler items. The test lists were preceded by 10 filler test pairs. These were not included in the analysis.

\section{Results}

Table 5 summarizes the results of Experiment 4. The mean proportions of choice appear in the two rows labeled "Observed." The $M S_{\mathrm{e}}=0.009$. A clear mirror pattern is present in both groups. The pattern of changes seen in Experiment 3 as a function of repetition appears again here. The standard choices show a change, and the null choices do not. The overall change in the null conditions is .02 . That change was tested and found not statistically significant $[t(115)=0.81]$. The pattern of movement schematized in Figure 5 holds.

\section{Discussion}

Experiment 4 replicated the findings of Experiment 3. The repetition of the study items increased the participants' ability to discriminate old items from new. This is seen in the increase in each of the standard forced-choice pairs from the single to the repetition group means. As in Experiment 3, however, the increase does not affect the null choice pairs. With repetition, the old and new distributions move away from the midpoint of the decision axis, but with little or no increase in the distances between $\mathrm{LN}$ and $\mathrm{HN}$ and between $\mathrm{HO}$ and LO. The pattern is, again, symmetric in that the lack of spread between the old distributions HO and LO is reflected in the lack of spread in the new distributions LN and HN.

To fit the theory to the data, the attention parameter was again allowed to vary. We used the same five parameters, $p$ (new), $n$ (low, 1), $n$ (low,2), $n$ (high, 1), and $n$ (high,2), to fit the 12 observed means. The fit is satisfactory $\left(r^{2}=.97\right)$. An examination of the attention parameter, $n(i, t)$, indicates that participants change their treatment of the high- and low-frequency words from the first to the sec- ond trial. As in Experiment 3, for the first presentation, the low-frequency attention parameter, $n$ (low, 1$)$, is higher than the high-frequency attention parameter, $n$ (high,1). For the second presentation, there is a drop in the values for both parameters, but, as in Experiment 3, the lowfrequency attention parameter, $n$ (low,2), drops farther than the high-frequency attention parameter, $n$ (high,2). The participants, when they viewed the low-frequency words during the second presentation, showed a sharper drop in attention than when they viewed high-frequency words.

To be sure of the results from repetition, we ran a third repetition experiment. We considered the possibility that the decrease in attention to the low-frequency words may have resulted from the relatively quick reappearance of these words within a similar context. In both repetition experiments, the second appearance of the study items occurred while the participants were performing the same task as they were during the first appearance. It is possible that if the second appearance occurred within a context dissimilar to the first, participants would not display the loss in attention indicated by the change in $n(i, t)$. The novelty of the second appearance might cause participants to maintain the level of attention elicited originally. If this were to happen, participants might show the full dispersion pattern seen in Experiment 2 rather than the restricted dispersion pattern seen in Experiments 3 and 4 . However, if the results were similar to those of the previous repetition experiments, then a case could be made that this pattern is a regular result of repetition.

\section{Experiment 5}

In Experiment 5, we used a repetition procedure designed to maintain the participants' attention across the two presentations and eliminate the differential reductions of $n(i, t)$. Our aim was to make the second presentation very different from the first presentation. This was done by having the test trial function as a second study trial.

In recognition memory experiments, participants are first presented words for study and are then tested. During test, though, participants have a further opportunity to learn the test items. In other words, the test also acts as a study trial. There is support for this statement in Runquist (1983). In that study, it was shown that a test trial increased performance on a second memory test.

In the design used here, the recognition test serves two purposes: It tests the results of the preceding study, and

Table 5

Mean Proportions of Choice for the Single and

Repeated Presentation of Words in Experiment 4

\begin{tabular}{|c|c|c|c|c|c|c|}
\hline \multirow[b]{2}{*}{ Condition } & \multicolumn{2}{|c|}{ Null Pairs } & \multicolumn{4}{|c|}{ Standard Pairs } \\
\hline & $P(\mathrm{HN}, \mathrm{LN})$ & $P(\mathrm{LO}, \mathrm{HO})$ & $P(\mathrm{HO}, \mathrm{HN})$ & $P(\mathrm{LO}, \mathrm{HN})$ & $P(\mathrm{HO}, \mathrm{LN})$ & $P(\mathrm{LO}, \mathrm{LN})$ \\
\hline \multicolumn{7}{|l|}{ Single } \\
\hline Observed & .65 & .63 & .66 & .71 & .76 & .79 \\
\hline Predicted & .65 & .63 & .67 & .73 & .75 & .78 \\
\hline \multicolumn{7}{|l|}{ Repeated } \\
\hline Observed & .66 & .64 & .71 & .80 & .81 & .89 \\
\hline Predicted & .66 & 63 & .74 & .81 & .82 & .86 \\
\hline
\end{tabular}

Note-Parameters: $p($ new $)=.09 ; N=1,000$ (preset); $n($ high, 1$)=35 ; n($ low, 1$)=53$; $n($ high, 2$)=20 ; n($ low, 2$)=24 ; r^{2}=.97$. There were 24 participants in each condition. 
it gives the participants a second chance to study the items but in a different context. A second recognition test follows the first test. In the second test, the old words, those seen in both the study list and first recognition test, are presented with a set of previously untested, new words. The second test measures the combined effect of the initial study and the first test trial.

\section{Method}

The participants were presented a list of words and instructed to study the items as they appeared. They were then given a twoalternative forced-choice recognition test to measure performance (single presentation). Following the completion of this test, the participants were given a second two-alternative forced-choice recognition test (repeated presentation). For the second test list, the old words were the words from the study list and, consequently, the old words from the first recognition test. The new words, however, were completely new and did not appear in either the previous study or test lists. The second test list, as with the first test list, was randomly created, with the only constraint being that all six choice conditions were equally represented for each subject. The experiment used two within-group independent variables: repetition and word frequency.

Participants. Twenty-four undergraduate students participated in the experiment.

Procedure. The experiment was run in three sections: study, first recognition test, and second recognition test. The study lists consisted of equal numbers of high-frequency and low-frequency words, which appeared on a computer monitor. Each word appeared alone, for $1,500 \mathrm{msec}$, with a $250-\mathrm{msec}$ ISI. The participants were told to study the words as they appeared and that a recognition test would follow.

After completing the study list, the participants were given two successive two-alternative forced-choice recognition tests. In the first test, the participants were instructed to choose the item of the pair that they thought appeared in the study list. In the second test, they were instructed to choose the item of the pair that had appeared in either the initial study list or the previous recognition test.

The experiment started with a practice session that consisted of a short, 10-word study list and two successive recognition tests. Each of the two practice recognition tests that followed consisted of 10 two-alternative forced-choice word pairs.

Materials. Five hundred forty items, half high-frequency words and half low-frequency words, formed the pool from which both old and new words were selected. From this pool, randomly selected and ordered study lists consisting of 90 high-frequency words and 90 low-frequency words, for a total of 180 words, were drawn. The test lists consisted of 180 pairs - 30 pairs in each of the six forced choice conditions. The old words for the first test list were those words that appeared in the study list; the new words were drawn randomly from the original pool of words and had not appeared previously. The old words for the second recognition test were the same as the old words in the first test. The new words for the second recognition test were the remaining 180 words in the original pool. They had not appeared in either the study list or the first recognition test. Both test lists were preceded by five filler pairs that were not included in the analysis. The study lists were preceded and followed by five filler items.

\section{Results}

The results replicated those of Experiments 3 and 4. Table 6 shows that both the single and the repetition groups display the full mirror effect and that repetition increases the discrimination between new and old items. The $M S_{\mathrm{e}}=0.005$. The effect of repetition is seen, however, only in the standard choices. As in Experiments 3 and 4, there was no increase in the null choice pairs (HN,LN and $\mathrm{LO}, \mathrm{HO}$ ). In the null choice pairs, there was a reversal in $P(\mathrm{LO}, \mathrm{HO})$, with the single-presentation condition greater than the repetition condition. In the new null pairs, $P(\mathrm{HN}, \mathrm{LN})$, the proportion of choice was larger in the repetition condition, but this difference was slight. A test again shows that the difference between single and repeated presentation for the null pairs combined was not statistically significant $[t(115)=0.40]$.

\section{Discussion}

The dispersion of the four underlying distributions follows the same pattern as that in Experiments 3 and 4. The two old and two new distributions move away from the center point of the decision axis, with no increase in the distances of $\mathrm{HN}$ from $\mathrm{LN}$ and of LO from HO. This is the third example of this pattern produced by repetition. Collectively, Experiments 3, 4, and 5 show that this pattern is a consistent and reliable product of repetition and word frequency. The symmetric pattern of movements is the one seen in Figure 5.

Five parameters, listed at the bottom of Table 6 $N$ (preset), $p$ (new), and four $n(i, t)$-were used again to fit the 12 observed means. The fit is satisfactory $\left(r^{2}=\right.$ .96). A pattern like that found in the preceding repetition experiments again emerges. Initially, the low-frequency attention parameter, $n$ (low, 1$)$, is much higher than the high-frequency attention parameter, $n($ high, 1 ). This difference disappears on the second test, and, indeed, the value of $n($ low,2) is lower than that of $n($ high,2). This indicates, again, that during the second presentation, participants reduce their processing of low-frequency words

Table 6

Mean Proportions of Choice for the Single- and Repeated-Presentation Conditions in Experiment 5

\begin{tabular}{|c|c|c|c|c|c|c|}
\hline \multirow[b]{2}{*}{ Condition } & \multicolumn{2}{|c|}{ Null Pairs } & \multicolumn{4}{|c|}{ Standard Pairs } \\
\hline & $P(\mathrm{HN}, \mathrm{LN})$ & $P(\mathrm{LO}, \mathrm{HO})$ & $P(\mathrm{HO}, \mathrm{HN})$ & $P(\mathrm{LO}, \mathrm{HN})$ & $P(\mathrm{HO}, \mathrm{LN})$ & $P(\mathrm{LO}, \mathrm{LN})$ \\
\hline \multicolumn{7}{|l|}{ Single } \\
\hline Observed & .61 & .65 & .70 & .77 & .76 & .81 \\
\hline Predicted & .64 & .63 & .70 & .77 & .77 & .81 \\
\hline \multicolumn{7}{|l|}{ Repeated } \\
\hline Observed & .63 & .64 & .75 & .79 & .84 & .87 \\
\hline Predicted & .64 & .63 & .76 & .82 & .82 & .86 \\
\hline
\end{tabular}

Note-Parameters: $p($ new $)=.09 ; N=1,000$ (preset); $n($ high, 1$)=40 ; n($ low, 1$)=58$; $n$ (high, 2$)=16 ; n($ low, 2$)=14 ; r^{2}=.96$. There were 24 participants in each condition. 
more than their processing of high-frequency words. This reduction causes the lack of spread between the two old distributions and between the two new distributions by reducing the outward movement of the low-frequency distributions. This occurs even when the items reappear in very different contexts, as in Experiment 5, where the old words first appeared in a study list and then in a recognition test.

\section{GENERAL DISCUSSION}

The experiments in this study tested the following predictions based on attention/likelihood theory: that the underlying distributions, both new and old, move symmetrically on the decision axis, and that increasing recognition performance should lead to an increase in the distances between the underlying distributions without changing their order. We used two methods to increase recognition memory: encoding tasks of different effectiveness, and repetition of stimulus items. The encoding tasks were expected to directly affect $n(i)$, the attention parameter of the theory. Repetition was not expected to alter $n(i)$ but, with the additional sampling opportunity of a repeated trial, to increase the proportion of features sampled and marked-that is, $p(i$, old $)$.

The encoding experiments showed the mirror pattern in both encoding task conditions, with the more effective encoding task producing greater recognition performance and a greater dispersion of the underlying distributions. The dispersion was complete in that all four distributions moved away from a central point and from each other. In particular, both the old distributions $\mathrm{LO}$ and $\mathrm{HO}$ and the new distributions $\mathrm{HN}$ and $\mathrm{LN}$ moved apart from each other in the more effective encoding condition. The increase in encoding affected both the items seen during study, as well as the new items (those not studied at all).

The repetition experiments produced a symmetric pattern different from that produced in the encoding experiments. Repetition consistently produced both the mirror pattern and an increase in recognition performance. The distances between the old (LO,HO) and new (HN,LN) distributions, however, did not increase from the first to the second trial, as with variation of encoding during study. This pattern was replicated across the three experiments with different procedures.

In all of the experiments, manipulations that affected the positions of the old distributions affected the positions of the new distributions as well. The symmetry of movement of the underlying distributions permit all these experiments to be fitted by attention/likelihood theory.

The experiments show again that variables that affect accuracy of recognition cause the underlying distributions, such as those of LN, HN, HO, and LO, to move symmetrically around a central point on the decision axis while maintaining the mirror pattern. Moreover, they show that this symmetrical movement can take a number of different forms. Glanzer et al. (1993) showed that forgetting, speed instructions during test, longer lists, and aging pro- duced concentering in which the underlying distributions move toward a central point, and each moves toward its neighbor. Kim and Glanzer (1995) showed that output interference produced a symmetrical movement of the underlying distributions toward a central point, but one in which the outer distributions, LN and LO, moved less than the inner distributions, $\mathrm{HN}$ and HO. In Experiments 1 and 2 here, we found for encoding the inverse of concentering, with the distributions moving away from a central point and each distribution moving away from its neighbor. Finally, in Experiments 3, 4, and 5, we found that, for repetition, the distributions moved away from a central point, but the inner distributions, $\mathrm{HO}$ and $\mathrm{HN}$, kept pace with the outer distributions, $\mathrm{LO}$ and $\mathrm{LN}$.

The point has been made earlier (Glanzer et al., 1993) that the global theories (Gillund \& Shiffrin, 1984; Hintzman, 1988; Murdock, 1982) cannot account for the mirror effect. They cannot, furthermore, account for the symmetric movement either centripetal, when accuracy of recognition goes down (Glanzer et al., 1991, 1993), or centrifugal, when accuracy of recognition increases.

In summary, we have demonstrated a general pattern of bilateral symmetric movement of underlying distributions that occurs with manipulations that increase recognition performance. Encoding differences gives one pattern of symmetric dispersion. Repetition gives another.

\section{REFERENCES}

BRENT, R. P. (1973). Algorithms for minimization without derivatives. Englewood Cliffs, NJ: Prentice-Hall.

Craik, F. I. M., \& Tulving, E. (1975). Depth of processing and the retention of words in episodic memory. Journal of Experimental Psychology: General, 104, 268-294.

Forbach, G. B., Stanners, R. F., \& Hochhaus, L. (1974). Repetition and practice effects in a lexical decision task. Memory \& Cognition, 2, 337-339.

GEGENFURTNER, K. R. (1992). PRAXIS: Brent's algorithm for function minimization. Behavior Research Methods, Instruments, \& Computers, 24, 560-564.

Gillund, G., \& SHiffrin, R. M. (1984). A retrieval model for both recognition and recall. Psychological Review, 91, 1-67.

GlanZER, M., \& ADAMS, J. K. (1985). The mirror effect in recognition memory. Memory \& Cognition, 13, 8-20.

Glanzer, M., \& ADAMS, J. K. (1990). The mirror effect in recognition memory: Data and theory. Journal of Experimental Psychology. Learning, Memory, \& Cognition, 16, 5-16.

Glanzer, M., Adams, J. K., \& Iverson, G. (1991). Forgetting and the mirror effect in recognition memory: Concentering of underlying distributions. Journal of Experimental Psychology: Learning, Memory, \& Cognition, 17, 81-93.

Glanzer, M., Adams, J. K., Iverson, G. J., \& Kim, K. (1993). The regularities of recognition memory. Psychological Review, 100, 546-567.

Glanzer, M., \& Bowles, N. (1976). Analysis of the word frequency effect in recognition memory. Journal of Experimental Psychology: Human Learning \& Memory, 2, 21-31.

Gorman, A. M. (1961). Recognition memory for nouns as a function of abstractness and frequency. Journal of Experimental Psychology, 61, 23-29.

Hintzman, D. L. (1988). Judgments of frequency and recognition memory in a multiple-trace memory model. Psychological Review, 95, 528-551.

KiM, K., \& Glanzer, M. (1993). Speed versus accuracy instructions, study time, and the mirror effect. Journal of Experimental Psychology: Learning, Memory, \& Cognition, 19, 638-652.

KIM, K., \& GLANZER, M. (1995). Intralist interference in recognition 
memory. Journal of Experimental Psychology: Learning, Memory, \& Cognition, 21, 1096-1107.

KUČERA, H., \& FrANCIS, W. N. (1967). Computational analysis of presentday American English. Providence RI: Brown University Press.

MURDOCK, B. B., JR. (1982). A theory for the storage of item and associative information. Psychological Review, 89, 609-626.

Paivio, A., Yuille, J. C., \& Madigan, S. A. (1968). Concreteness, imagery, and meaningfulness values for 925 nouns. Journal of Experimental Psychology Monographs, 76(1, Pt. 2).

Piéron, H. (1914). Recherches sur les lois de variation des temps de latence sensorielle en fonction des intensités excitatrices [Research on the laws of variation in sensory latency as a function of excitatory intensities]. L'Année Psychologique, 20, 17-96.

Pí́ron, H. (1920). Nouvelles recherches sur l'analyse du temps de latence sensorielle et sur la loi qui relie ce temps a l'intensité de l'ex- citation [New research on the analysis of sensory latency and on the law that relates this time to the intensity of excititation]. L'Année Psychologique, 22, 58-142.

RuNQUiST, W. N. (1983). Some effects of remembering on forgetting. Memory \& Cognition, 11, 641-650.

Scarborough, D. L., Cortese, C., \& Scarborough, H. S. (1977). Frequency and repetition effects in lexical memory. Journal of Experimental Psychology: Human Perception \& Performance, 3, 1-17.

SCHNEIDER, W. (1988). Micro Experimental Laboratory: An integrated system for IBM PC compatibles. Behavior Research Methods, Instruments, \& Computers, 20, 206-217.

(Manuscript received September 17, 1995; revision accepted for publication July 18, 1996.) 